\title{
SETS OF POSTULATES FOR THE LOGIC OF PROPOSITIONS*
}

\author{
BY \\ B. A. BERNSTEIN
}

1. Introduction. Boole $\nmid$ created the mathematical sciences now known as the logic of classes and the logic of propositions. $\ddagger$ Peirce $\S$ and Schröder\| have perfected these sciences. The logic of classes now rests on rigorous, postulational foundations. Various sets of postulates, with consistency, independence, and sufficiency proved, have been worked out for that logic. I But no rigorous postulate sets exist for the logic of propositions. ${ }^{* *}$ The object of this paper is to supply such sets for this logic.

My starting point is Schröder's analysis of the logic. $\dagger \dagger$ The propositional logic is the body of facts concerning the truth or falsity of propositions $a, b, c, \cdots$ and their combinations. Every proposition $a$ is either true or else it is false. If true, we write $a=1$; if false, we write $a=0$. Every fact in this logic, symbolically expressed, is also a fact in the logic of classes consisting of the elements 0,1 ; and conversely. With Schröder, therefore, I define the logic of propositions to be a two-element logic of classes. It is the algebra of truth-values $0,1 . \sharp$

* Presented to the Society, San Francisco Section, June 19, 1925; received by the editors in October, 1925.

† George Boole, An Investigation of the Laws of Thought, London, 1854.

$\ddagger$ Boole develops these logics as theories of "primary" and "secondary" propositions. Boole's theories, as improved by Peirce and Schröder, may be called, after Sheffer, boolean algebras.

$\S$ C. S. Peirce. For a list of Peirce's writings see M. R. Cohen, Journal of Philosophy, Psychology, and Scientific Methods, vol. 13 (1916), p. 733.

II E. Schröder. See his Vorlesungen über die Algebra der Logik, Leipzig, vol. 1, 1890; vol. 2, part 1, 1891 ; vol. 2, part 2, edited by E. Müller, 1905. Vol. 2, part 1, will be referred to in the later footnotes simply as Vorlesungen.

I See, e. g., E. V. Huntington, these Transactions, vol. 5 (1904), p. 288; H. M. Sheffer, ibid., vol. 14 (1913), p. 481 ; B. A. Bernstein, ibid., vol. 17 (1916), p. 50.

** Boole states inaccurately (Laws of Thought,p.159) that the formal laws underlying his theory of primary propositions are identical with those underlying his theory of secondary propositions. Schröder is mistaken in thinking (Vorlesungen, pp. 51-52) that by adding to any postulate set for the class logic a postulate to the effect that the logic consists of two elements, a set of independent postulates will thereby be obtained for the propositional logic. Whitehead and Russell's set of primitives for their "theory of deduction" (Principia Mathematica, vol. 1, pp. 94-101) is imperfect. The authors fail, among other things, to give a satisfactory discussion of the independence of the primitive propositions (see a later footnote).

†† See Vorlesungen, $\$ \S 21-31$.

$\ddagger \ddagger$ Schröder and other mathematical logicians do not distinguish between the symbols $<,+, \times$ that belong to the propositional logic (either as primitive ideas or as ideas defined by means of the primitive ideas) and the notions "implies," "or," "and" respectively that are outside the logic. Thus if $a, b, c$ are elements of our logic and " $<$ " the relation of "implication" belonging to the logic, then we have the proposition

(i) If $a<b$ and $b<c$, then $a<c$. 
Four sets of postulates are obtained. If we denote by $K$ a class of elements and by,$+ \times, \mid$ the operations of logical addition, multiplication, rejection* respectively, the undefined notions in the sets are

$$
K,+, \times ; K, \mid ; K,+; \quad K \text {. }
$$

(For convenience $a \times b$ will be written $a \cdot b$ or $a b$.) The postulates in these sets are in number respectively five, four, three, one. As will be seen, the first two sets are more than a mere selection from corresponding postulate sets for the general logic of classes. The fourth set is quite novel. It consists of a single postulate involving a single undefined notion, the notion of class.

For each set, the complete existential theory $\dagger$ of the postulates is established, Tables I, II, III exhibiting the existential theories of the first three sets. In these tables the signs,$+ \times$ at the head of the columns are enclosed in circles, in order to distinguish them from the arithmetic,$+ \times$ employed in the concrete systems found in the body of the tables. In the

Or, writing "implies" for " $<$ ", we have

(ii) If " $a$ implies $b$ " and " $b$ implies $c$," then " $a$ implies $c$. "

But Schröder (Vorlesungen, p. 28) would write (ii) in the form

(iii)

$$
[(a<b) \times(b<c)]<(a<c)
$$

where the "if . . . then" and "and" of (ii), which are outside the logic, are replaced by the symbols " $<$ " and " $X$ " of the logic. It is this confusion between the symbols of the logic of propositions and notions outside the logic that leads Schröder (Vorlesungen, pp. 45-48) to go through a lengthy explanation of why the duality principle that exists in the logic of classes breaks down in the logic of propositions when, as a matter of fact, it does not break down!

It is the failure to differentiate properly between the symbols of the propositional logic and notions outside the logic that causes Whitehead and Russell in their Principia, vol. 1, to hold (p. 94) that the theory of propositions is more fundamental than the theory of classes and (p. 95, footnote) that the recognized methods of proving the independence of a set of postulates are inapplicable to the primitive propositions of the logic of propositions. If $P$ is the set of postulates of any mathematical science $\Sigma$, and $Q$ the body of propositions deducible from $P$, then, as Russell has well said somewhere, the science $\Sigma$ is simply the proposition " $P$ implies $Q . "$ Now the relation "implies" in this proposition is outside $\Sigma$, even if $\Sigma$ is the logic of propositions in which a relation "<" of "implication" is involved. Indeed, the notions "proposition," "negation," etc. and the syllogism, the law of contradiction,etc. are all used in unsymbolized forms in deducing these very laws symbolically from an appropriate set of postulates for the logic of propositions. The unsymbolized forms of these notions and laws belong to a general logic, a sub-logic, underlying the logic that is receiving symbolic treatment. And there is no circularity in all this. On the contrary, one must recognize a sub-logic as underlying any symbolic logic, if he is to avoid circularity. The recognition of this sub-logic is essentially the observance of Russell's own principle, used by him so effectively in the solution of paradoxes, the principle that no collection may contain the collection itself as a member. And so, proper differentiation between the symbols and laws of a propositional logic and the corresponding ideas and laws of its sub-logic would lead to the exclusion from the set of primitive ideas and primitive propositions for the propositional logic of the Principia notions such as "assertion" and propositions such as "Anything implied by a true elementary proposition is true." The resulting set of primitives would not be in the least more fundamental for logic than a set of primitives for the logic of classes, and the usual methods of proving independence would be applicable to it.

* For the concrete meaning of this operation see H. M. Sheffer, loc. cit.

† See E. H. Moore, Introduction to a Form of General Analysis, New Haven Colloquium, Yale University Press, p. 82. 
concrete systems, an operation followed by "mod 3" (or " $\bmod 2$ ") denotes the least positive integer obtained from the operation by dropping multiples of 3 (or 2).

2. Set I. Postulates in terms of addition and multiplication. The following postulate set for the logic of propositions is modelled after Huntington's first set* of ten postulates for the general logic of classes. It leaves undefined class $K$ and operations,$+ \times$, and it exhibits the law of duality in the logic. Note that postulates 3,4 are not found in Huntington's set.

1. There is a $K$-element 0 such that $0+a=a$ for every $K$-element $a$.

2. There is a $K$-element 1 such that $1 \cdot a=a$ for every $K$-element $a$.

3. If an element 0 of postulate 1 exists, then $0 \cdot a=0$ for every $K$ element $a$.

4. If an element 1 of postulate 2 exists, then $1+a=1$ for every $K$ element $a$.

5. $K$ consists of two elements.

3. Sufficiency of Set I. Propositions 6-14 below, derived from Set I, together with postulates 1, 2, 5, give Huntington's first set of postulates for the general logic of classes, and hence prove the sufficiency of Set I for the logic of propositions.

6. $0 \neq 1$.

For if $0=1$, take (by 5) a $K$-element $a$ not 0 ; then $a=1 \cdot a=0 \cdot a=0$, by 2 , hyp., 3 .

7. 0 and 1 are unique.

For $K$ has no elements other than 0,1 , by 5,6 . And $1+0=1 \neq 0$, by 4,$6 ; 0 \cdot 1=0 \neq 1$, by 3,6 .

8. $a+b$ is a $K$-element whenever $a, b$ are $K$-elements.

For $0+b=b, 1+b=1$, by 1,4 .

9. $a b$ is a $K$-element whenever $a, b$ are $K$-elements.

For $0 \cdot b=0,1 \cdot b=b$, by 3,2 .

10. $a+b=b+a$.

For $0+1=1=1+0$, by 1,4 .

11. $a b=b a$.

For $0 \cdot 1=0=1 \cdot 0$, by 3,2 .

12. $a+b c=(a+b)(a+c)$.

For $0+b c=b c=(0+b)(0+c)$, by 1,$1 ; 1+b c=1=1 \cdot 1=(1+b)(1+c)$, by $4,2,4$.

13. $a(b+c)=a b+a c$.

For $0(b+c)=0=0+0=0 \cdot b+0 \cdot c$, by $3,1,3 ; 1(b+c)=b+c=1 b+1 c$, by 2,2 .

* E. V. Huntington, loc. cit. 
14. For every $K$-element $a$ there is a $K$-element $a^{\prime}$ such that $a+a^{\prime}=1$, $a a^{\prime}=0$.

For $0+1=1,0 \cdot 1=0$, by 1,$3 ; 1+0=1,1 \cdot 0=0$, by 4,2 .

Therefore, $0^{\prime}=1,1^{\prime}=0$.

4. Complete existential theory of Set I. Table I below exhibits the complete existential theory of the postulates of Set I. System 32 proves the consistency of the postulates and systems 16, 24, 28, 30, 31 prove their (ordinary) independence. The non-existence (indicated by blanks) of systems having the characters $(- \pm- \pm \pm),( \pm- \pm- \pm)$ is in accordance with the evident facts that postulate 3 cannot be contradicted if postulate 1 is denied, and that postulate 4 cannot be contradicted if postulate 2 is denied.

Table I

Existential Theory of Set I

\begin{tabular}{|c|c|c|c|c|}
\hline No. & Character & $K$ & $a \oplus b$ & $a \odot b$ \\
\hline 1 & $(-----)$ & - & - & - \\
\hline 2 & $(---+)$ & - & - & 一 \\
\hline 3 & $(---+-)$ & - & 一 & - \\
\hline 4 & $(---++)$ & - & - & 一 \\
\hline 5 & $(--+--)$ & - & - & - \\
\hline 6 & $(--+-+)$ & - & - & - \\
\hline 7 & $(--++-)$ & 0 & $0 / 0$ & $0 / 0$ \\
\hline 8 & $(--+++)$ & 0,1 & $0 / 0$ & $0 / 0$ \\
\hline 9 & $(-+---)$ & - & - & - \\
\hline 10 & $(-+--+)$ & 一 & - & - \\
\hline 11 & $(-+-+-)$ & 一 & - & - \\
\hline $\begin{array}{l}12 \\
13\end{array}$ & $\begin{array}{l}(-+-++) \\
(-++-)\end{array}$ & $\overline{0}$ & $\overline{0 / 0}$ & $\overline{0}$ \\
\hline 14 & $(-++-+)$ & 0,1 & 0 & $a b$ \\
\hline 15 & $(-+++)$ & $0,1,2$ & 1 & $a b \bmod 3$ \\
\hline 16 & $(-++++)$ & 0,1 & 1 & $a b$ \\
\hline 17 & $(+----)$ & - & - & - \\
\hline 18 & $(+---+)$ & - & - & - \\
\hline 19 & $(+--+-)$ & 0 & 0 & $0 / 0$ \\
\hline 20 & $(+--++)$ & 0,1 & $a b$ & $\mathbf{0}$ \\
\hline 21 & $(+-t--)$ & - & - & - \\
\hline 22 & $(+-t-+)$ & - & - & - \\
\hline 23 & $(+-t+-)$ & $0,1,2$ & $a b \bmod 3$ & 1 \\
\hline 24 & $(t-t+t)$ & 0,1 & $a b$ & 1 \\
\hline 25 & $(++---)$ & $0,1,2$ & $a+b \bmod 3$ & $a+b+2 \bmod 3$ \\
\hline 26 & $(t+--t)$ & 0,1 & $a b+b \bmod 2$ & \\
\hline 27 & $(++-+-)$ & $0,1,2$ & $\begin{array}{r}2 a^{2} b+a+b \bmod 3 \\
a b+a+b \bmod 2\end{array}$ & $\begin{array}{l}a+b+2 \bmod 3 \\
a+b+1 \bmod 2\end{array}$ \\
\hline 28 & $(++-++)$ & 0,1 & $\begin{array}{l}a b+a+b \bmod 2 \\
a+b+2 \bmod 3\end{array}$ & $\begin{array}{c}a+b+1 \bmod 2 \\
2 a^{2} b+a+b \bmod 3\end{array}$ \\
\hline $\begin{array}{l}29 \\
30\end{array}$ & $\begin{array}{l}(+++--) \\
(t+t-t)\end{array}$ & $\begin{array}{c}0,1,2 \\
0,1\end{array}$ & $\begin{array}{l}a+b+2 \bmod 3 \\
a+b+1 \bmod 2\end{array}$ & $a b+a+b \bmod 2$ \\
\hline 31 & $(t+t+-)$ & 0 & 0 & 0 \\
\hline 32 & $(t+t+t)$ & 0,1 & $a b+a+b \bmod 2$ & $a b$ \\
\hline
\end{tabular}


5. Set II. Postulates in terms of rejection. A set of independent postulates sufficient for the logic of propositions, expressed in terms of class $K$ and Sheffer's operation of rejection "|", is the set $A-D$ below. Note how much simpler this set is than the corresponding sets given by Sheffer* and by the writer $i$ for boolean algebras in general.

A. $a \mid b$ is a unique $K$-element whenever $a, b$ are $K$-elements.

Definition. $a^{\prime}=a \mid a$.

B. $a^{\prime} \neq a$ whenever $a, a \mid a$ are $K$-elements.

C. $a|b=b| a$ whenever $a, b, a|b, b| a$ are $K$-elements, and $a \neq b$.

$D$. $K$ consists of two elements.

6. Sufficiency of Set II. Theorems $E-K$ following, derived from Set II, prove the sufficiency of this set for our logic of propositions, since propositions $\mathrm{A}, \mathrm{D}, \mathrm{J}, \mathrm{K}$ give the set of postulates previously proved by the writer $\ddagger$ sufficient for boolean algebras. In the proofs of the theorems reference to postulate A will generally be omitted.

E. $\left(a^{\prime}\right)^{\prime}=a$.

By A, D, B.

F. $a\left|a^{\prime}=a^{\prime}\right| a=b \mid b^{\prime}$.

For $a\left|a^{\prime}=a^{\prime}\right| a$, by $\mathrm{B}, \mathrm{C}$; if $b \neq a$, then $b=a^{\prime}$, by $\mathrm{D}, \mathrm{B}$, hence $b \mid b^{\prime}=$ $a^{\prime}\left|\left(a^{\prime}\right)^{\prime}=a^{\prime}\right| a$, by $\mathrm{E}$.

Definitions. $0=a \mid a^{\prime} ; 1=0^{\prime}$.

G. $1^{\prime}=0$.

By E.

H. $a|0=0| a=a^{\prime}$.

For $0 \mid 0=0^{\prime}$, by def.; $1|0=1| 1^{\prime}=0=1^{\prime}$, by $\mathrm{G}$, def., G.

I. $a|1=1| a=0$.

For $0 \mid 1=1^{\prime}=0$, by $H, G ; 1 \mid 1=1^{\prime}=0$, by def., G.

J. $(b \mid a) \mid\left(b^{\prime} \mid a\right)=a$.

For $(0 \mid a)\left|\left(0^{\prime} \mid a\right)=a^{\prime}\right|(1 \mid a)=a^{\prime} \mid 0=\left(a^{\prime}\right)^{\prime}=a$, by H, def., I, H, E; (1 $\left.\mid a\right) \mid\left(1^{\prime} \mid a\right)$ $=0|(0 \mid a)=0| a^{\prime}=\left(a^{\prime}\right)^{\prime}=a$, by I, G, H, H, E.

K. $a^{\prime} \mid\left(b^{\prime} \mid c\right)=\left[\left(b \mid a^{\prime}\right) \mid\left(c^{\prime} \mid a^{\prime}\right)\right]^{\prime}$.

For $0^{\prime}\left|\left(b^{\prime} \mid c\right)=1\right|\left(b^{\prime} \mid c\right)=0$, by def., I; $\left[\left(b \mid 0^{\prime}\right) \mid\left(c^{\prime} \mid 0^{\prime}\right)\right]^{\prime}=\left[(b \mid 1) \mid\left(c^{\prime} \mid 1\right)\right]^{\prime}=$ $(0 \mid 0)^{\prime}=\left(0^{\prime}\right)^{\prime}=0$, by def., I, def., E. Also $1^{\prime}\left|\left(b^{\prime} \mid c\right)=0\right|\left(b^{\prime} \mid c\right)=\left(b^{\prime} \mid c\right)^{\prime}$, by G, $\mathrm{H} ;\left[\left(b \mid 1^{\prime}\right) \mid\left(c^{\prime} \mid 1^{\prime}\right)\right]^{\prime}=\left[(b \mid 0) \mid\left(c^{\prime} \mid 0\right)\right]^{\prime}=\left[b^{\prime} \mid\left(c^{\prime}\right)^{\prime}\right]^{\prime}=\left(b^{\prime} \mid c\right)^{\prime}$, by G, H, E.

7. Complete existential theory of Set II. Table II following establishes the complete existential theory of Set II. The postulates of this set are

* Loc. cit.

$\dagger$ Loc. cit.

$\ddagger$ Loc. cit. 
seen to be completely independent, ${ }^{*}$ system 16 proving the consistency of the postulates and systems $8,12,14,15$ proving their (ordinary) independence.

Table II

Existential Theory of Set II

\begin{tabular}{c|c|c|c||c|c|c|c}
\hline \hline No. & Character & $K$ & $a \mid b$ & Co. & Character & $K$ & $a \mid b$ \\
\hline 1 & $(----)$ & $0,1,2$ & $b+0 / b$ & 9 & $(+--))$ & $0,1,2$ & $b$ \\
2 & $(---+)$ & 0,1 & $b+0 /(a b+a+b) \bmod 2$ & 10 & $(+--+)$ & 0,1 & $b$ \\
3 & $(--+-)$ & $0,1,2$ & $1+0 / b$ & 11 & $(+-+-)$ & 0 & 0 \\
4 & $(--++)$ & 0,1 & $1+0 /(a b+a+b) \bmod 2$ & 12 & $(+-++)$ & 0,1 & $a b+a+b \bmod 2$ \\
5 & $(-+--)$ & $0,1,2$ & $b+2+0 / b \bmod 3$ & 13 & $(++--)$ & $0,1,2$ & $b+1 \bmod 3$ \\
6 & $(-+-+)$ & 0,1 & $a+1+0 /(a b+a+b) \bmod 2$ & 14 & $(++-+)$ & 0,1 & $a+1 \bmod 2$ \\
7 & $(-++-)$ & 0 & $0 / 0$ & 15 & $(+++-)$ & null & - \\
8 & $(-+++)$ & 0,1 & $0+0 /(a b+a+b) \bmod 2$ & 16 & $(++++)$ & 0,1 & $a b+a+b+1 \bmod 2$ \\
\hline
\end{tabular}

8. Set III. Postulates in terms of addition. Corresponding to Huntington's set of nine postulates $\dagger$ for the general logic of classes expressed in terms of class $K$ and operation of addition we may use for the logic of propositions the three postulates $\alpha-\gamma$ following.

$\alpha$. There is a $K$-element 0 such that $0+a=a$ for every $K$-element $a$.

$\beta$. There is a $K$-element 1 such that $1+a=1$ for every $K$-element $a$.

$\gamma$. $K$ consists of two elements.

9. Sufficiency of Set III. We have, first,

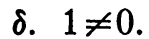

For otherwise, if $x$ be any element, $x=0+x=1+x=1$, by $\alpha, \beta$. But this is contrary to $\gamma$.

Definitions. $0^{\prime}=1 ; 1^{\prime}=0 ; a b=\left(a^{\prime}+b^{\prime}\right)^{\prime}$.

The sufficiency of Set III for the logic of propositions then follows from Theorems $\eta, \zeta, \theta$ below, since $\zeta, \theta$ are postulates 2,3 respectively of Set $\mathrm{I}$. $\eta$. $\left(a^{\prime}\right)^{\prime}=a$.

For $\left(0^{\prime}\right)^{\prime}=1^{\prime}=0$ by def., def.; $\left(1^{\prime}\right)^{\prime}=0^{\prime}=1$, by def., def.

ร. $1 a=a$.

For $1 a=\left(1^{\prime}+a^{\prime}\right)^{\prime}=\left(0+a^{\prime}\right)^{\prime}=\left(a^{\prime}\right)^{\prime}=a$, by def., def., $\alpha, \eta$.

$\theta$. $0 \cdot a=0$.

For $0 \cdot a=\left(0^{\prime}+a^{\prime}\right)^{\prime}=\left(1+a^{\prime}\right)^{\prime}=1^{\prime}=0$, by def., def., $\beta$, def.

10. Complete existential theory of Set III. The complete existential theory of Set III is given by Table III following. The set is seen to be completely independent.

* E. H. Moore, loc. cit.

† E. V. Huntington, loc. cit. 
Table III

Existential Theory of Set III

\begin{tabular}{c|c|c|c||c|c|c|c}
\hline \hline No. & Character & $K$ & $a \oplus b$ & No. & Character & $K$ & $a \oplus b$ \\
\hline 1 & --- & 0 & $0 / 0$ & 5 & +-- & $0,1,2$ & $b$ \\
2 & --+ & 0,1 & $b+1 \bmod 2$ & 6 & +-+ & 0,1 & $b$ \\
3 & -+- & $0,1,2$ & 1 & 7 & ++- & 0 & 0 \\
4 & -++ & 0,1 & 1 & 8 & +++ & 0,1 & $a b+a+b \bmod 2$ \\
\hline
\end{tabular}

11. Set IV. A one-postulate set. The following set consists of the single postulate $\mathrm{P}$, in which the notion of class $K$ is the only notion left undefined. The operations having the properties of logical addition and logical multiplication are both defined by means of $\mathbf{P}$.

Postulate P. $K$ consists of two elements.

DeFinitions.

1. Denote the two $K$-elements by 0,1 .

2. $a+b: 0+0=0,0+1=1,1+0=1,1+1=1$.

3. $a b: \quad 0 \cdot 0=0,0 \cdot 1=0,1 \cdot 0=0,1 \cdot 1=1$.

Postulates $1-5$ of Set $I$ then follow, so that postulate $P$ is sufficient for our logic.

12. A new principle in postulate theory. The procedure employed in $\S 11$ makes evident the following new principle in postulate theory: If $a$ system is finite, a single postulate, expressed in terms of the single undefined notion of class, is sufficient for the system.

UNIVERSITY OF CAIIfORNIA, Berkeley Calif 